\title{
Study of parameters for evaluating flow reduction with stents in a sidewall aneurysm phantom model
}

\author{
Chang Ho Yu and Tae Kyu Kwon* \\ Division of Biomedical Engineering, Chonbuk National University, 567 Baekje-daero, Deokjin-gu, \\ Jeonju-si, Jeonbuk 561-756, Republic of Korea
}

\begin{abstract}
The effect of stent design parameters such as porosity, pore density, number of strands, and strut angle to the artery were studied in vitro using particle image velocimetry (PIV). Five mesh stents were implanted into a sidewall aneurysm model. The flow features in a sidewall aneurysm silicone phantom model were investigated at a Reynolds number of 300 . It was found that the lowest porosity stent had the best value for velocity and vorticity reduction in an aneurysm pocket. The stent with higher pore density had a tendency to decrease the mean and maximum velocities, but it was not superior to the effects of porosity. In addition, investigation of the evaluation system confirmed that higher stent strut angles to the parent artery had a tendency to lower mean velocity, as shown by PIV and CFD. However, this effect was relatively smaller compared to porosity and pore density. Our evaluation system suggested the best combinations of parameters for the development of an ideal stent would be lower porosity, higher pore density, and higher strut angle. The results obtained in this study indicated that our evaluation system could be useful for various purposes related to evaluation of endovascular interventional devices.
\end{abstract}

Keywords: Particle Image Velocimetry (PIV), stent, sidewall aneurysm, porosity, pore density

\section{Introduction}

A cerebral aneurysm refers to a cerebrovascular pathological abnormality, which occurs commonly near the end of a vascular bifurcation and looks like a pocket [1]. An intravascular treatment using the catheter for stent insertion has proven to be useful for aneurysm treatment as a minimally invasive treatment technique [2-4]. However, the mechanical properties of a stent for cerebral aneurysm treatment depend on superior deliverability, which is an important determinant and is also a final determining factor of clinical usefulness. Physicians need to be aware of the differences in model-specific stent movements for optimal clinical results [5]. Particle Image Velocimetry (PIV) using different stents in the same blood flow conditions was used to demonstrate the effectiveness of medical device for these

\footnotetext{
${ }^{*}$ Corresponding author: Tae Kyu Kwon, Division of Biomedical Engineering, Chonbuk National University, 567 Baekjedaero, Deokjin-gu, Jeonju-si, Jeonbuk 561-756, Republic of Korea. Tel.: +82-63-270-4066; Fax: +82-63-270-2247; E-mail: kwon10@jbnu.ac.kr.
}

0959-2989/14/\$27.50 @ 2014 - IOS Press and the authors. 
intravascular treatments. PIV is an optical method of flow visualization. It is used to examine the related properties of the fluid and instantaneous velocity measurements.

Hemodynamic studies for blood flow patterns using PIV in states of steady flow and pulsatile flow with a stent and no stent win an aneurysm phantom model were performed [6,7]. Previous researchers have sought to understand the hemodynamics, but a full understanding has not been reached because the hemodynamics is influenced by aneurysm placement and expansion. Nevertheless, previous researchers considered that rapid blood flow velocity and shear stress on the aneurysm wall will be able to create a hemodynamic atmosphere for rupture and injury of a blood vessel.

Various strategies have been studied on the basis of a mesh type stent [8-11]. A mesh type stent reduced the wall shear stress on the aneurysm wall, and it was reported that high shear stress on the stent surface was represented in ideal and patient-specific aneurysm models. However, in most previous studies, the subject of mesh type stent design (i.e., based on the variable stent design), did not include a variable hemodynamic evaluation.

The purpose of this study was to evaluate hemodynamic changes based on stent design variables, such as porosity, pore density, number of strands, and stent strut shape, and then develop an estimating system for stents using PIV.

\section{Experimental methods}

\subsection{Structure of stent and silicone sidewall aneurysm phantom model}

Five mesh stents (TaeWoong Medical Co., Ltd., Korea) were tested in this study. The porosity ranges of the stents were $100 \%, 80 \%, 74 \%, 71 \%$ and $64 \%$. Table 1 shows the detail dimensions. The stents all had different design parameters, i.e., porosity, pore density, number of strands, and stent strut shape. The stents consisted basically of a mesh-work of rhombus-shape openings, as shown in Figures 1(a) and 1(b). Figures 1(b) and 1(c) also show scanning electron microscope (SEM) images of the stents, to provide an easy understanding of the structure of stents used in this experiment.

In this study, steady flow conditions were forced on a rigid model. We chose the simple sidewall aneurysm model, since this geometry of aneurysm is one of the simplest ones, and it provides better realization of the flow conditions of the poiseuille in this experiment. This is very important, because it would be difficult to evaluate the stent efficiency with real flow conditions using aneurysm models with a different geometry. Figures 1(d) and 1(e) show the silicone sidewall aneurysm phantom model.

Table 1

Dimension of stent samples

\begin{tabular}{|l|l|l|l|l|l|}
\hline & Stent 1 & Stent 2 & Stent 3 & Stent 4 & Stent 5 \\
\hline Porosity (\%) & 80 & 74 & 64 & 71 & 71 \\
\hline $\begin{array}{l}\text { Pore density } \\
\text { (Picks per inch) }\end{array}$ & 75 & 93 & 123 & 133 & 158 \\
\hline (Picks per inch) & & & & & \\
\hline Strands & 32 & 32 & 32 & 48 & 64 \\
\hline Strut diameter $(\mathrm{mm})$ & 0.038 & 0.038 & 0.038 & 0.030 & 0.025 \\
\hline Angles $(\alpha, \beta)$ & $124^{\circ}, 56^{\circ}$ & $140^{\circ}, 40^{\circ}$ & $148^{\circ}, 32^{\circ}$ & $140^{\circ}, 40^{\circ}$ & $140^{\circ}, 40^{\circ}$ \\
\hline $\mathrm{H} \times \mathrm{L}(\mathrm{mm})$ & $0.64 \times 0.36$ & $0.65 \times 0.27$ & $0.54 \times 0.16$ & $0.44 \times 0.17$ & $0.34 \times 0.15$ \\
\hline
\end{tabular}




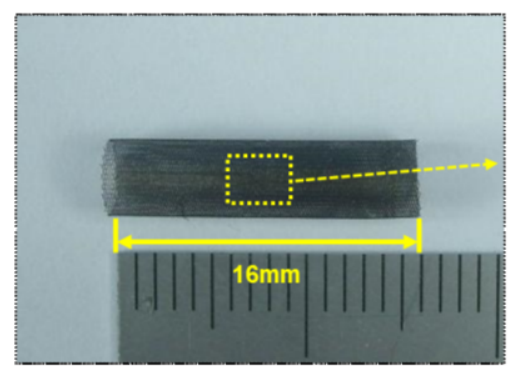

(a)

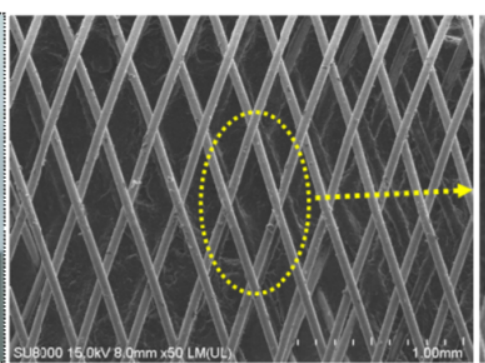

(b)

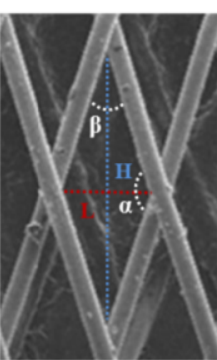

(c)

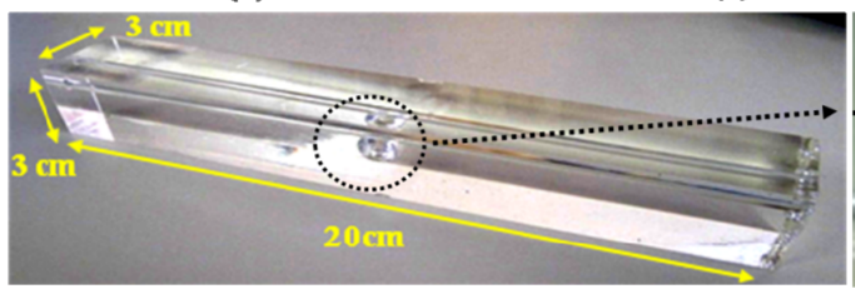

(d)

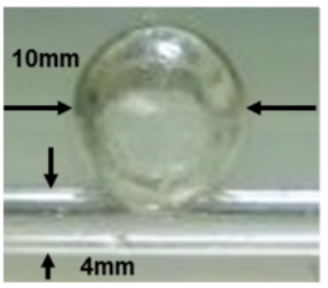

(e)

Fig. 1. (a) Mesh-type stent sample. (b) Scanning electron microscope (SEM) image of the stent. (c) Image enlargement of the stent. (d) Photo of the silicone sidewall aneurysm phantom model. (e) Image enlargement of the sphere.

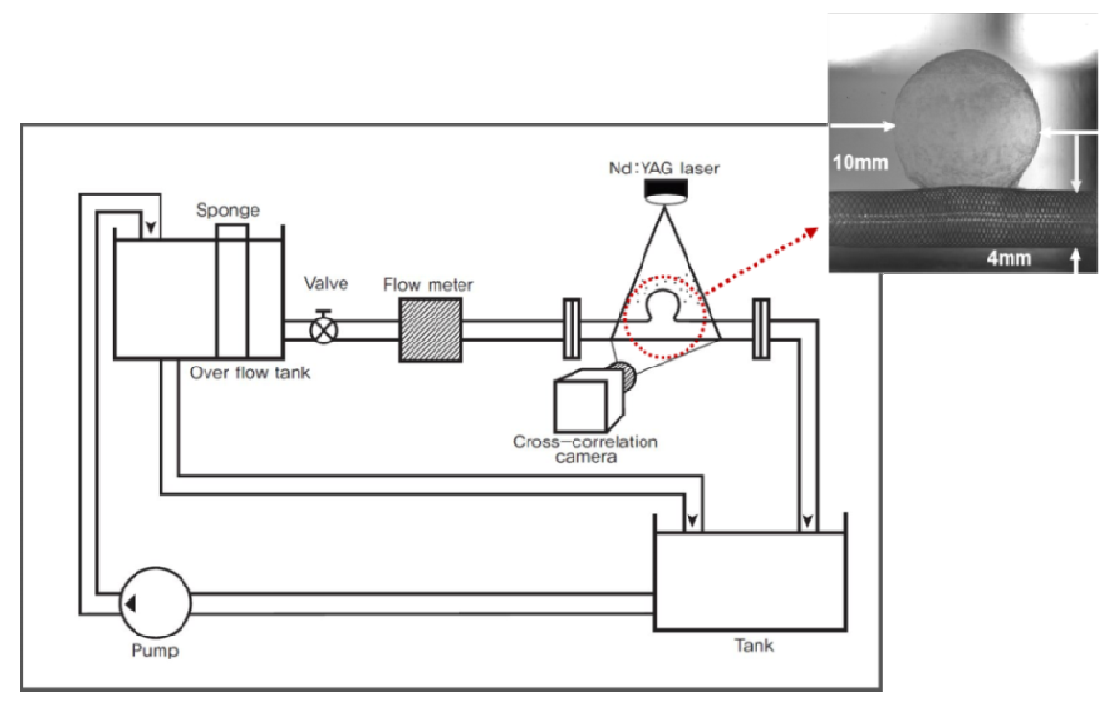

Fig. 2. Schematic of overall flow system.

\subsection{The overall flow system}

A silicone phantom model of an optimized sidewall aneurysm was constructed. This aneurysm phantom model was made with a $4 \mathrm{~mm}$ diameter in the neck plane, a $10 \mathrm{~mm}$ core box for aneurysm dome height, and silicone with straight stiffness (Figure 2). This phantom model was connected to blood flow loop with an elastic polyvinyl chloride tube and the same vascular resistance was applied at the outlet. The corresponding refractive index was determined to be 1.408 at $24.4^{\circ} \mathrm{C}$ with glycerol at $57.3 \mathrm{wt} \%$. The working fluid of $1.144 \times 103 \mathrm{~kg} / \mathrm{m} 3$ density and $6.99 \times 10-3 \mathrm{~kg} / \mathrm{m} 3$ dynamic viscosity 
was circulated through the model. The solution contains the $17.41 \mu \mathrm{m}$ cross-linked polystyrene powder. Steady flow was used to stream through the blood vessel from the main vessel just before the aneurysm. The steady flow streamed through the loop using a pump.

Flow velocity data were obtained using a PIV system (Flowmaster PIV system, LAVISION GmbH, Germany). The PIV system diagram is shown in Figure 2. A frame transfer CCD camera was used to obtain images of $1024 \times 1024$ pixel resolution. A $532 \mathrm{~nm} \mathrm{Nd:YAG} \mathrm{laser} \mathrm{was} \mathrm{used} \mathrm{to} \mathrm{lighten} \mathrm{the} \mathrm{par-}$ ticles in the inner space of model with a thickness of $0.5 \mathrm{~mm}$ flat beam. To pass the particle fluorescence while blocking the laser reflection from the deployed stent, an optical low-pass filter was used. [12]. Measurements were made in both the xy plane and the xz plane in all cases. The center of the aneurysm was 0 plane, and 9 planes, from 4 to -4 , were analyzed for $x y$. Under the parent vessel was a plane, and 9 planes, from a to $\mathrm{i}$, were analyzed for $\mathrm{xz}$ (Figure 3 ).

\section{Results}

\subsection{Effectiveness of stent porosity}

We compared the effects of stents with differing porosities $(80,74,71,64 \%)$ and no stent $(100 \%$ porosity) using the silicone phantom model of a sidewall aneurysm. The flow velocity reduction changed depending on stent porosity. Inflow in this work means the flow immediately before the passage of the stent. The average and maximum velocities with no stent were greater than those of the stent velocity in all planes (Figure 4). There was a direct correlation between stent type used and blood flow modification in the aneurysm. Variable changes, such as porosity or stent wire dimensions, had great influences on the flow. The porosity range used in this study was lower than in a previous study (64\%). Maintaining the stent porosity in the $60 \%$ range is most effective in terms of slowing the movement of the blood flow.

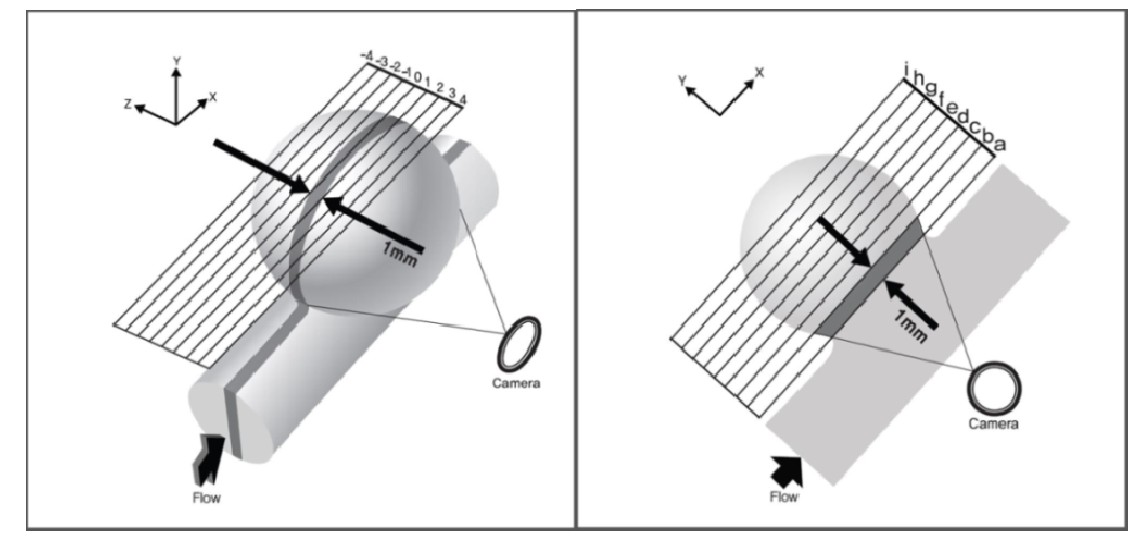

Fig. 3. Definition of laser irradiation position in $x y$ and $x z$ planes.

Figure 4 suggests that stent porosity is one of the most important factors related to velocity reduction. The results indicate that lower porosity stents had a tendency for higher velocity reduction rates than higher porosity stents. It also showed that the factor of stent porosity alone was not sufficient to uniquely explain the reduction in flow velocity in the neck area and pocket of the aneurysm. To over- 
come the shortcomings of stent porosity, our study also took into account how the shape of the stent struts and stent strut angle to the parent artery affect the flow patterns in an aneurysm pocket.

\subsection{Effectiveness of stent pore density and number of strands}

The two important characteristics of our mesh stent design were the porosity and the pore density, which is defined as the number of pores per unit surface area [13]. We modified the stent pore density, which was defined as the number of struts per unit inch in our experimental study, used the term of PPI (Picks per inch) for definition. All five configurations had an open device diameter of $4 \mathrm{~mm}$ and an identical surface pattern; pore densities of the mesh-shaped stents 1,2, 3, 4 and 5 were approximately 75, 93, 123, 133 and 158 PPI, respectively. However, all stents were made up of different numbers of wires. This is termed as the number of strands. Stent 1, 2, 3 were made up of 32 strands (Number of wire), stent 4 was made up of 48 strands, and stent 5 was made up of 64 strands. As shown in Figure 5, stents with a higher pore density and number of strands had a tendency to have decreased mean and maximum velocities in the aneurysm pocket. However, the stent with the lowest porosity (64\%) was more useful for reducing the mean and maximum velocities, compared to the stent with the highest pore density.

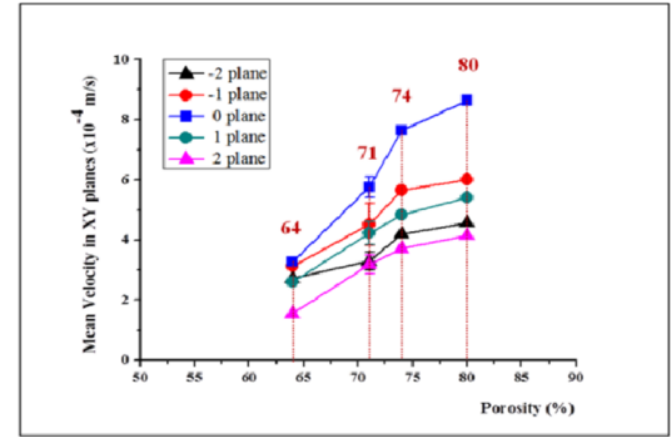

(a)

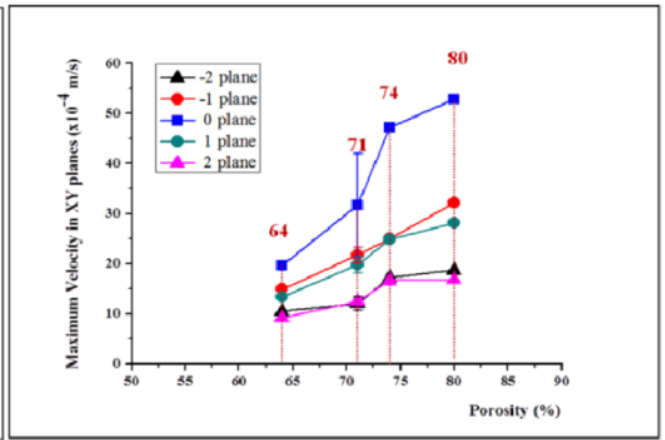

(b)

Fig. 4. (a) Change in mean velocities of the xy plane: model with stent 1 (porosity: $80 \%$ ), stent 2 (porosity: 74\%), stent 3 (porosity: 64\%), stent 4 (porosity: $71 \%$ ), and stent 5 (porosity: $71 \%$ ). (b) Change in maximum velocities of xy plane: model with stent 1 (porosity: 80\%), stent 2 (porosity: 74\%), stent 3 (porosity: 64\%), stent 4 (porosity: 71\%), and stent 5 (porosity: $71 \%)$.

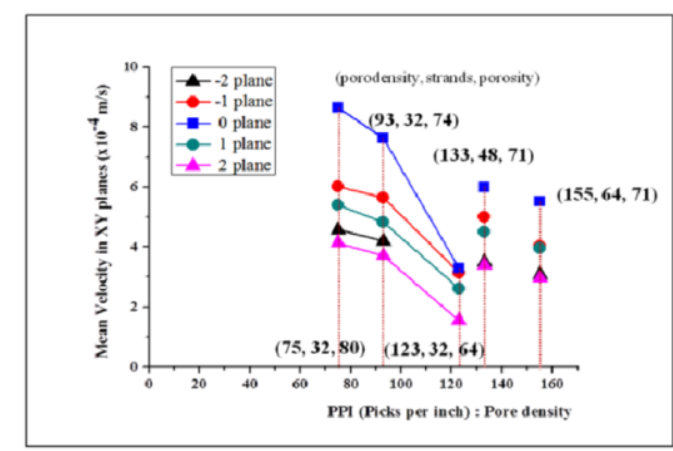

(a)

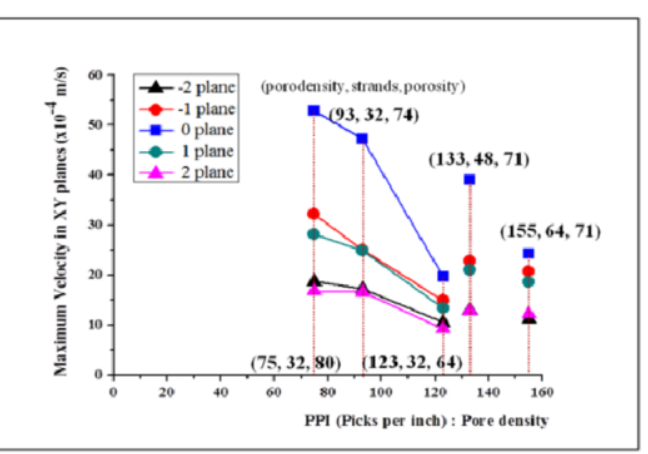

(b)

Fig. 5. (a) The mean velocity according to pore density; (b) The maximum velocity according to pore density. 


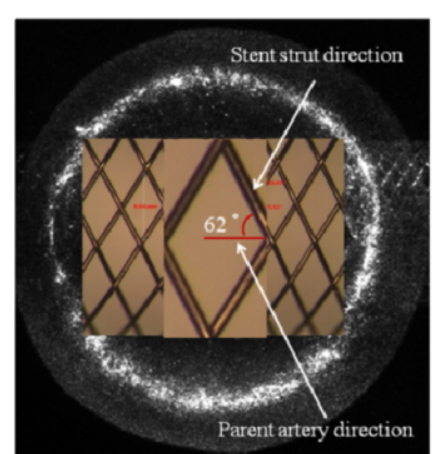

(a)

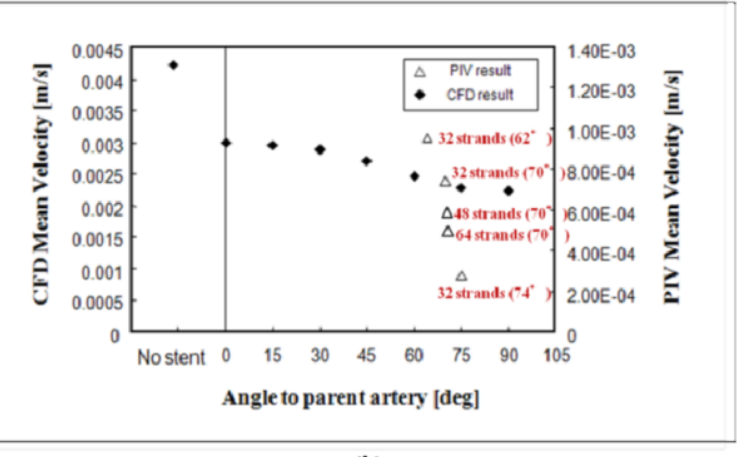

(b)

Fig. 6. (a) Stent strut angle to parent artery; (b) Validation of the mean velocity reduction between CFD and PIV.

\subsection{Effectiveness of stent strut angle to parent artery}

To evaluate the effect of stent strut shape, we considered the stent strut angle to the parent artery, which is defined as the angle between the direction of the stent strut and the parent artery, as another important parameter in our experimental study (Figure 6). This evaluation also focused on the concept of flow velocity reduction rate to identify the stent efficiency. All five configurations had an open device diameter of $4 \mathrm{~mm}$, and identical surface patterns; stent strut angle to the parent artery of meshshaped stents 1, 2, 3, 4 and 5 were approximately 62, 70, 74, 70 and 70 degrees, respectively. Figure 6(b) shows the validation of the mean velocity between CFD and PIV. X axis represents the angel to parent artery from 0 to 105 degree. Y axis represents the mean velocity in CFD and PIV, respectively. The mean velocity according to the stent strut angle to the parent artery was measured. The mean velocity reduction was then compared by considering computational fluid dynamics (CFD) and PIV. As shown in Figure 6(b), higher stent strut angles to the parent artery had a tendency to lower the mean velocity in PIV and CFD [14].

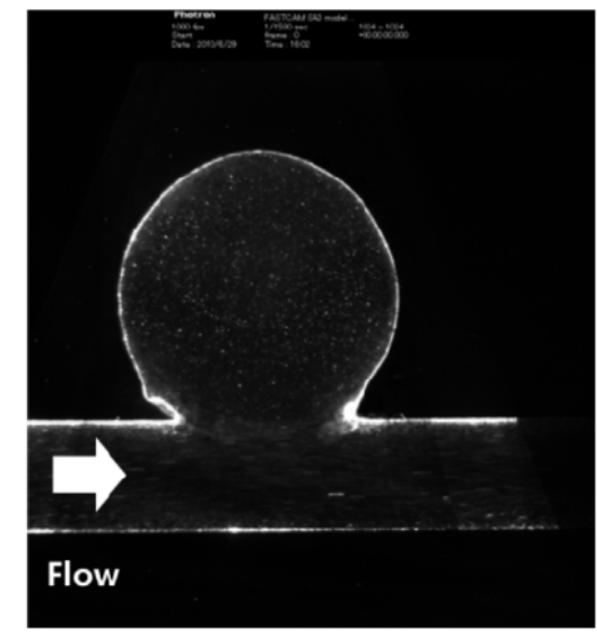

(a)

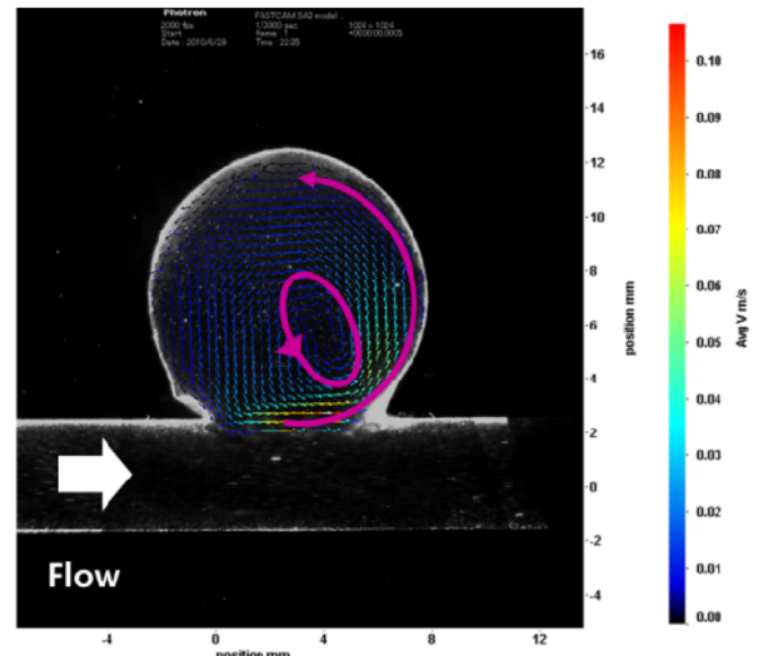

(b)

Fig. 7. (a) Laser light sheet method can obtain which particles are dispersed in the flow field; (b) In vitro results showing velocity vector plots at a 0 plane in XY plane with no stent (porosity: 100\%). 


\section{Discussion}

We describe the characteristics of our newly developed evaluation system. We found that several parameters affected the behavior of flow reduction. Performance analyses with and without stents were conducted using experimental and computational simulations. As a result of fundamental experiments and computer simulations, it was found that stent can be hidden in a sidewall aneurysm [15]. Wakhloo et al. [16] performed a visualization of flow to investigate the effect of stent porosity in terms of changes in flow pattern in the aneurysm using laser-induced fluorescence with a rhodamine dye. However, almost all previous studies failed to clarify the design problem of mesh-type stents: i.e., evaluating changes in hemodynamics based on variable stent design. The current study addresses this.

We developed a system for stent estimation by evaluating the changes in hemodynamics based on the stent design variables, such as new mesh-type stents and porosity, pore density, the number of strands, and stent strut form in this research. Hemodynamic characteristics of the aneurysm were assessed using PIV. The geometry of a sidewall aneurysm was selected for the system. This geometric structure is one of the simplest, but it provides a good realization of the Poiseuille flow conditions of viscosity. This is important because stent efficiency in a state of real flow is difficult to evaluate using an aneurysm model with different geometric structures.

As shown in Figure 7, a silicone aneurysm phantom model with no stent shows the recirculation vortex of the aneurysm; the center is located near the distal cervical region. Decreased flow and flow velocity in the aneurysm pocket were observed when inserting a stent with low porosity. The velocity was reduced by up to $98 \%$ due to the low porosity. However, the one factor of porosity was insufficient to fully describe the impact of the stent. To overcome these problems, we also evaluated the velocity reduction due to the mesh stent design, by analyzing the pore density, number of strands, and stent strut angle to the parent artery. Stents with a higher pore density and number of strands had a similar tendency, to decrease the mean and maximum velocities in the aneurysm pocket.

Porosity was found to be a major factor. The stent with the lowest porosity $(64 \%)$ demonstrated the most effective reduction in the mean and maximum velocities, being even more effective than the stent with the highest pore density ( 158 picks per inch). It was also observed that a higher number of strands in the stent affected the mean and maximum velocity reduction. Finally, higher stent strut angles to the parent artery showed a tendency to lower the mean velocity in PIV and CFD.

\section{Conclusion}

We evaluated our new system in terms of several parameters. The effectiveness of the stent was determined by evaluating changes in hemodynamics based on stent design parameters, such as porosity, pore density, number of strands, and stent strut shape.

Our evaluation system suggested that the best combination of parameters for the development of an ideal stent would be lower porosity, higher pore density, and higher strut angle. The results obtained in this study indicated that our evaluation system may be useful for various purposes related to the evaluation of endovascular interventional devices. 


\section{Acknowledgment}

This research was supported by Basic Science Research Program through the National Research Foundation of Korea (NRF) funded by the Ministry of Education (2013R1A1A2011620).

\section{References}

[1] Y.H. Kim, X. Xu and J.S. Lee, The effect of stent porosity and strut shape on saccular aneurysm and its numerical analysis with lattice boltzman method, Annals of Biomedical Engineering 38 (2010), 2274-2292.

[2] G.W. Stone, J.W. Moses, S.G. Ellis, J. Schofer, K.D. Dawkins and M. Morice, Safety and efficacy of sirolimus- and paclitaxel-eluting coronary stents, New England Journal of Medicine 356 (2007), 998-1008.

[3] B. Lagerqvist, S.K. James, U. Stenestrand, J. Lindback, T. Nilsson and L. Wallentin, Long-term outcomes with drugeluting stents versus bare-metal stents in Sweden, New England Journal of Medicine 356 (2007), 1009-1019.

[4] L. Mauri, W.H. Hsieh, J.M. Massaro, K.K.L. Ho, R. D’Agostino and D.E. Cutlip, Stent thrombosis in randomized clinical trials of drug-eluting stents, New England Journal of Medicine 356 (2007), 1020-1029.

[5] W. Schmidt, P. Lanzer, P. Behrens, L.D.T. Topoleski and K.P. Schmitz, A comparison of the mechanical performance characteristics of seven drug-eluting stent system, Catheterization and Cardiovascular Interventions 73 (2009), 350-360.

[6] S.C.M. Yu and J.B. Zhao, A steady flow analysis on the stented and non-stended sidewall aneurysm models, Medical Engineering and Physics 21 (1999), 133-141.

[7] S.C.M. Yu and J.B. Zhao, A particle image velocimetry study on the pulsatile flow characteristics in straight tubes with an asymmetric bulge, Proceedings of the Institution of Mechanical Engineers 214 (1999), 655-671.

[8] M. Aenis, A.P. Stancampiano, A.K. Wakhloo and B.B. Lieber, Modeling of flow in a straight stented and nonstented side wall aneurysm model, Journal of Biomechanical Engineering 119 (1997), 206-212.

[9] G.R. Stuhne and D.A. Steinman, Finite-element modeling of the hemodynamics of stented aneurysms, Journal of Biomechanical Engineering 126 (2004), 382-387.

[10] J.R. Cebral and R. Lohner, Efficient simulation of blood flow past complex endovascular devices using an adaptive embedding technique, IEEE Transactions on Medical Imaging 24 (2005), 468-476.

[11] K. Bando and S.A. Berger, Research on fluid-dynamic design criterion of stent used for treatment of aneurysms by means of computational simulation, Computational Fluid Dynamics Journal 11 (2003), 527-531.

[12] C.H. Yu, K. Matsumoto, S. Shida, D.J. Kim and M. Ohta, A steady flow analysis on a cerebral aneurysm model with several stents for new stent design using PIV, Journal of Mechanical Science and Technology 26 (2012), 1333-1340.

[13] B.B. Lieber, A.P. Stancampiano and A.K. Wakhloo, Alteration models by stenting: influence of stent porosity, Annals of Biomedical Engineering 25 (1997), 460-469.

[14] K. Okuno, T. Nakayama, D.A. Rufenacht and M. Ohta, Effect of stent strut position of flow pattern in an ideal cerebral aneurysm model, Proceedings of the 21th Bioengineering Conference, 2008, 61-62.

[15] M.P. Mark, M.K. Dake, G.K. Steinberg, A.M. Norbash and B. Lane, Stent placement of arterial and venous cerebrovascular disease: preliminary clinical experience, Reaiology 191 (1994), 441-446.

[16] C. Sadasivan, L. Cesar, J. Seong, A. Rakian, M.Q. Hao, F. Tio, A.K. Wakhloo and B.B. Lieber, An original flow diversion device for the treatment of intracranial aneurysms: evaluation in the rabbit elastase-induced model, Stroke 40 (2009), 952-958. 